\title{
Theta Constants Associated with the Cyclic Triple Coverings of the Complex Projective Line Branching at Six Points
}

By

\author{
Keiji Matsumoto*
}

\begin{abstract}
Let $\psi$ be the period map for a family of the cyclic triple coverings of the complex projective line branching at six points. The symmetric group $S_{6}$ acts on this family and on its image under $\psi$. In this paper, we give an $S_{6}$-equivariant expression of $\psi^{-1}$ in terms of fifteen theta constants.
\end{abstract}

\section{$\S 1 . \quad$ Introduction}

Let $C(\lambda)$ be the cyclic triple covering of the complex projective line $\mathbb{P}^{1}$ branching at six points $\lambda_{1}, \ldots, \lambda_{6}$ :

$$
C(\lambda): w^{3}=\prod_{i=1}^{6}\left(z-\lambda_{i}\right) .
$$

The moduli space of such curves with a homology marking can be regarded as the configuration space $\Lambda$ of ordered six distinct points on $\mathbb{P}^{1}$, which is defined by

$$
G L_{2}(\mathbb{C}) \backslash\left\{\lambda=\left(\lambda_{i j}\right) \in M(2,6)|\lambda\langle i j\rangle=| \begin{array}{cc}
\lambda_{1 i} & \lambda_{1 j} \\
\lambda_{2 i} & \lambda_{2 j}
\end{array} \mid \neq 0\right\} /\left(\mathbb{C}^{*}\right)^{6} .
$$

Note that the symmetric group $S_{6}$ naturally acts on $\Lambda$. It is shown in [15] that the map

$$
\iota: \Lambda \ni \lambda \mapsto\left[\ldots, y_{\langle i j ; k l ; m n\rangle}, \ldots\right]=[\ldots, \lambda\langle i j\rangle \lambda\langle k l\rangle \lambda\langle m n\rangle, \ldots] \in \mathbb{P}^{14}
$$

Communicated by K. Saito, November 15, 2000.

2000 Mathematics Subject Classification(s): Primary, 32N15; Secondary 11F55, 14J15, $32 \mathrm{G} 20$.

* Division of Mathematics, Graduate School of Science, Hokkaido University, Sapporo 0600810, Japan. 
is an $S_{6}$-equivariant embedding and that its image is an open subset of $Y$ defined by linear and cubic equations.

The normalized period matrix $\Omega$ of $C(\lambda)$ with a homology marking belongs to the Siegel upper half space $\mathbb{S}^{4}$ of degree 4 . By our assignment of the homology marking, $\Omega$ can be identified with an element of 3-dimensional complex ball $\mathbb{B}^{3}=\left\{x \in \mathbb{P}^{3} \mid{ }^{t} \bar{x} H x<0\right\}$, where $H=\operatorname{diag}(1,1,1,-1)$. In this way, we get a multi-valued map $\psi: \Lambda \rightarrow \mathbb{B}^{3} \subset \mathbb{S}^{4}$, which is called the period map. Results in [3], [6] and [13] imply that the image of $\psi$ is an open dense subset of $\mathbb{B}^{3}$, the monodromy group of $\psi$ is the principal congruence subgroup $\Gamma(1-\omega)$ of level $(1-\omega)$ of $\Gamma=\left\{g \in G L_{4}(\mathbb{Z}[\omega]) \mid{ }^{t} \bar{g} H g=H\right\}$, and that the inverse of $\psi$ is single valued.

In this paper, we express the inverse of the period map $\psi$ in terms of fifteen theta constants. More precisely, for the two isomorphisms $\psi: \Lambda \rightarrow$ $\psi(\Lambda) / \Gamma(1-\omega)$ and $\iota: \Lambda \rightarrow \iota(\Lambda) \subset Y \subset \mathbb{P}^{14}$, we present an isomorphism $\Theta: \psi(\Lambda) / \Gamma(1-\omega) \rightarrow \iota(\Lambda)$ such that the following diagram commutes:

$$
\begin{array}{cl}
\Lambda & \stackrel{\psi}{\longrightarrow} \psi(\Lambda) / \Gamma(1-\omega) \\
\iota \downarrow & \Theta \swarrow \\
\iota(\Lambda) \subset Y \subset \mathbb{P}^{14} . &
\end{array}
$$

The map $\Theta$ is given by the ratio of the cubes of the fifteen theta constants on $\mathbb{S}^{4}$ which are invariant under the action of $\Gamma(1-\omega)$ embedded in $S p(8, \mathbb{Z})$. Since it is easy to express the inverse of $\iota^{-1}$, the map $\Theta$ gives the inverse of $\psi$. In particular, there are linear and cubic relations among the cubes of fifteen theta constants which coincide with the defining equations of $Y \subset \mathbb{P}^{14}$.

It is known that $\Gamma /\left\langle\Gamma(1-\omega),-I_{4}\right\rangle$ is isomorphic to $S_{6}$, which naturally acts on $\psi(\Lambda) / \Gamma(1-\omega)$. The period map $\psi$ is $S_{6}$-equivariant. By considering the action $S_{6} \simeq \Gamma /\left\langle\Gamma(1-\omega),-I_{4}\right\rangle$ on the fifteen theta characteristics, we label fifteen theta constants as $(i j ; k l ; m n)$, where $\{i, j, k, l, m, n\}=\{1, \ldots, 6\}$. Then it turns out that the diagram (1) is $S_{6}$-equivariant.

An explicit expression of $\psi^{-1}$ is given in [5]. We want to know the combinatorial structure of $\psi^{-1}$ in order to study the inverse of the period map from a family of smooth cubic surfaces to the 4-dimensional complex ball $\mathbb{B}^{4}$ in [1]. This inverse map is constructed in [9].

For a 2-dimensional subfamily of ours defined by $\lambda_{5}=\lambda_{6}$, the period map and its inverse are studied in [11] and [12]. 


\section{$\S 2$. Configuration Space $\Lambda$ of Six Points on $\mathbb{P}^{1}$}

Let $M(m, n)$ be the set of complex $(m \times n)$ matrices. We define the configuration space $\Lambda$ of ordered six distinct points on the complex projective line $\mathbb{P}^{1}$ as

$$
\Lambda=G L_{2}(\mathbb{C}) \backslash M^{\prime}(2,6) /\left(\mathbb{C}^{*}\right)^{6},
$$

where

$$
M^{\prime}(2,6)=\left\{\lambda=\left(\lambda_{i j}\right) \in M(2,6)|\lambda\langle k l\rangle=| \begin{array}{cc}
\lambda_{1 k} & \lambda_{1 l} \\
\lambda_{2 k} & \lambda_{2 l}
\end{array} \mid \neq 0(1 \leq k \neq l \leq 6)\right\},
$$

and $G L_{2}(\mathbb{C})$ and $\left(\mathbb{C}^{*}\right)^{6}$ (regarding as the group of $(6 \times 6)$ diagonal matrices) act naturally on $M^{\prime}(2,6)$ from the left and right, respectively. Note that we regard the column vectors of $\lambda \in M^{\prime}(2,6)$ as the homogeneous coordinates of six points on $\mathbb{P}^{1}$ and the action of $G L_{2}(\mathbb{C})$ as the projective transformation. Six distinct points $\lambda_{1}, \ldots, \lambda_{6}$ on $\mathbb{C}$ are expressed by an element of $\Lambda$ by $(2 \times 6)$ matrix

$$
\lambda=\left(\begin{array}{cccccc}
1 & 1 & 1 & 1 & 1 & 1 \\
\lambda_{1} & \lambda_{2} & \lambda_{3} & \lambda_{4} & \lambda_{5} & \lambda_{6}
\end{array}\right)
$$

By normalizing $\left(\lambda_{1}, \lambda_{2}, \lambda_{3}\right)$ as $(\infty, 0,1)$, matrices of the form

$$
\left(\begin{array}{cccccc}
0 & 1 & 1 & 1 & 1 & 1 \\
1 & 0 & 1 & \ell_{1} & \ell_{2} & \ell_{3}
\end{array}\right), \quad \ell_{i} \neq 0,1, \ell_{j}(1 \leq i<j \leq 3)
$$

represent $\Lambda$.

We define a map $\iota$ from $\Lambda$ to the 14 -dimensional projective space $\mathbb{P}^{14}$ by

$$
\iota: \Lambda \ni \lambda \mapsto\left[\ldots, y_{\langle i j ; k l ; m n\rangle}, \ldots\right]=[\ldots, \lambda\langle i j\rangle \lambda\langle k l\rangle \lambda\langle m n\rangle, \ldots] \in \mathbb{P}^{14}
$$

where $\lambda$ is a $(2 \times 6)$ matrix represent of an element of $\Lambda$ and projective coordinates of $\mathbb{P}^{14}$ are labeled by $I=\langle i j ; k l ; m n\rangle(\{i, j, k, l, m, n\}=\{1, \ldots, 6\}, i<$ $j, k<l, m<n)$. Since the image $\iota(\lambda)$ is invariant under the actions $G L_{2}(\mathbb{C})$ and $\left(\mathbb{C}^{*}\right)^{6}$, this map is well defined. We use the following convention

$$
y_{\langle i j ; k l ; m n\rangle}=y_{\langle k l ; i j ; m n\rangle}=y_{\langle i j ; m n ; k l\rangle}=-y_{\langle j i ; k l ; m n\rangle} \cdot
$$

The image $\iota(\Lambda)$ is studied in [15], it is described as the following.

Fact 2.1. The closure $Y=\overline{\iota(\Lambda)}$ of $\iota(\Lambda)$ is a subvariety of $\mathbb{P}^{14}$ defined by the linear and cubic equations

$$
\begin{gathered}
y_{\langle i j ; k l ; m n\rangle}-y_{\langle i j ; k m ; l n\rangle}+y_{\langle i j ; k n ; l m\rangle}=0 \\
y_{\langle i j ; k l ; m n\rangle} y_{\langle i k ; j n ; l m\rangle} y_{\langle i m ; j l ; k n\rangle}=y_{\langle i j ; k n ; l m\rangle} y_{\langle i k ; j l ; m n\rangle} y_{\langle i m ; j n ; k l\rangle} .
\end{gathered}
$$


We define $\hat{\Lambda}$ as the compactification of $\Lambda$ isomorphic to $Y$.

\section{$\S 3 . \quad$ Period Matrix of $C$}

Let $C=C(\lambda)$ be the cyclic triple covering of $\mathbb{P}^{1}$ branching at six distinct points $\lambda_{i}^{\prime} \mathrm{s}$ :

$$
C(\lambda): w^{3}=\prod_{i=1}^{6}\left(z-\lambda_{i}\right)
$$

this curve is of genus 4 . Let $\rho$ be the automorphism of $C$ defined by

$$
\rho: C \ni(z, w) \mapsto(z, \omega w) \in C,
$$

where $\omega=(-1+\sqrt{-3}) / 2$. We give a basis of the vector space of holomorphic 1-forms on $C$ as follows

$$
\varphi_{1}=\frac{d z}{w}, \quad \varphi_{2}=\frac{d z}{w^{2}}, \quad \varphi_{3}=\frac{z d z}{w^{2}}, \quad \varphi_{4}=\frac{z^{2} d z}{w^{2}} .
$$

For a fixed $\lambda$ such that $\lambda_{i} \in \mathbb{R}, \lambda_{1}<\ldots<\lambda_{6}$, we take a symplectic basis $\left\{A_{1}, \ldots, A_{4}, B_{1}, \ldots, B_{4}\right\}$ of $H_{1}(C, \mathbb{Z})$ (i.e., $A_{i} \cdot A_{j}=B_{i} \cdot B_{j}=0, B_{i} \cdot A_{j}=\delta_{i j}$ ) such that

$$
\rho\left(B_{i}\right)=A_{i}(i=1,2,3), \quad \rho\left(B_{4}\right)=-A_{4},
$$

see Figure 1.

Put

$$
\left(\begin{array}{c}
\int_{A_{i}} \varphi_{j} \\
\int_{B_{i}} \varphi_{j}
\end{array}\right)_{i, j}=\left(\begin{array}{l}
\Omega_{A} \\
\Omega_{B}
\end{array}\right)
$$

Let $\varphi$ be the normalized basis of vector space of holomorphic 1-forms so that $\Omega_{B}$ becomes $I_{4}$. Note that the normalized period $\Omega=\Omega_{A} \Omega_{B}^{-1}$ belongs to the Siegel upper half space $\mathbb{S}^{4}$ of degree 4 . The next proposition shows that $\Omega$ can be expressed in terms of

$$
x={ }^{t}\left(x_{1}, \ldots, x_{4}\right)={ }^{t}\left(\int_{A_{1}} \varphi_{1}, \ldots, \int_{A_{4}} \varphi_{1}\right) .
$$

Proposition 3.1. We have

$$
\begin{aligned}
\Omega & =\omega\left[I_{4}-(1-\omega)\left(x{ }^{t} x H\right) /\left({ }^{t} x H x\right)\right] H=\omega\left[H-(1-\omega)\left(x{ }^{t} x\right) /\left({ }^{t} x H x\right)\right] \\
& =\left(\begin{array}{ccccc}
\omega & & & \\
& \omega & & \\
& & \omega & \\
& & & \\
& &
\end{array}\right)-\frac{\sqrt{-3}}{x_{1}^{2}+x_{2}^{2}+x_{3}^{2}-x_{4}^{2}}\left(\begin{array}{llll}
x_{1} x_{1} & x_{1} x_{2} & x_{1} x_{3} & x_{1} x_{4} \\
x_{2} x_{1} & x_{2} x_{2} & x_{2} x_{3} & x_{2} x_{4} \\
x_{3} x_{1} & x_{3} x_{2} & x_{3} x_{3} & x_{3} x_{4} \\
x_{4} x_{1} & x_{4} x_{2} & x_{4} x_{3} & x_{4} x_{4}
\end{array}\right),
\end{aligned}
$$

where $H=\operatorname{diag}(1,1,1,-1)$ and ${ }^{t} \bar{x} H x<0$. 


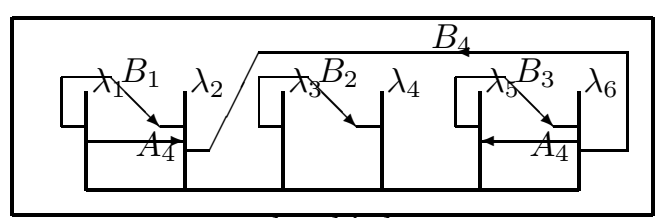

the third $z$-space

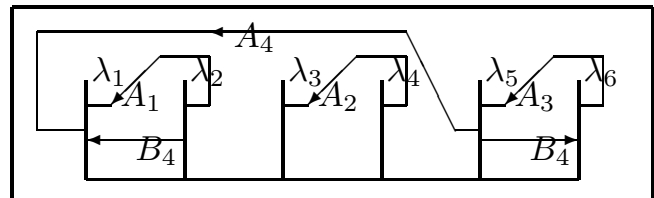

the second $z$-space

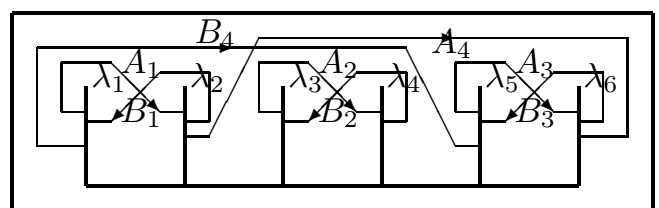

the first $z$-space

Figure 1. Basis of $H_{1}(C, \mathbb{Z})$

Proof. Put $\Omega_{A}=(x, b, c, d)$; by (2) and (3), $\Omega_{B}$ can be expressed as

$$
\Omega_{B}=\left(\omega H x, \omega^{2} H b, \omega^{2} H c, \omega^{2} H d\right)=\omega^{2} H \Omega_{A}+\left(\omega-\omega^{2}\right) H(x, O) .
$$

We have

$$
\Omega^{-1}=\Omega_{B} \Omega_{A}^{-1}=\omega^{2} H+\left(\omega-\omega^{2}\right) H(x, O) \Omega_{A}^{-1} .
$$

Put

$$
\Omega_{A}^{-1}=\left(\begin{array}{c}
\xi \\
*
\end{array}\right), \quad \xi=\left(\xi_{1}, \ldots, \xi_{4}\right)
$$

note that

$$
\xi x=\sum_{i=1}^{4} \xi_{i} x_{i}=1 .
$$

We have

(4) $\quad H(x, O) \Omega_{A}^{-1}=H x \xi=\frac{1}{\xi x}\left(\begin{array}{cccc}x_{1} \xi_{1} & x_{1} \xi_{2} & x_{1} \xi_{3} & x_{1} \xi_{4} \\ x_{2} \xi_{1} & x_{2} \xi_{2} & x_{2} \xi_{3} & x_{2} \xi_{4} \\ x_{3} \xi_{1} & x_{3} \xi_{2} & x_{3} \xi_{3} & x_{3} \xi_{4} \\ -x_{4} \xi_{1} & -x_{4} \xi_{2} & -x_{4} \xi_{3} & -x_{4} \xi_{4}\end{array}\right)$, 
which must be symmetric. Thus we have

$$
x_{i} \xi_{j}=x_{j} \xi_{i}(1 \leq i<j \leq 3), \quad x_{i} \xi_{4}=-x_{4} \xi_{i}(i=1,2,3) .
$$

By eliminating $\xi_{i}$ in (4), we have

$$
H(x, O) \Omega_{A}^{-1}=\left(H x{ }^{t} x H\right) /\left({ }^{t} x H x\right) .
$$

Then

$$
\Omega^{-1}=\omega^{2} H\left[I_{4}-\left(1-\omega^{2}\right)\left(x{ }^{t} x H\right) /\left({ }^{t} x H x\right)\right] .
$$

It is easy to see that

$$
\left[I_{4}-\left(1-\omega^{2}\right)\left(x{ }^{t} x H\right) /\left({ }^{t} x H x\right)\right]^{-1}=I_{4}-(1-\omega)\left(x{ }^{t} x H\right) /\left({ }^{t} x H x\right),
$$

we have

$$
\Omega=\omega\left[I_{4}-(1-\omega)\left(x^{t} x H\right) /\left({ }^{t} x H x\right)\right] H .
$$

The imaginary part of $\Omega$ is $\sqrt{3} / 2$ times

$$
H-x{ }^{t} x /\left({ }^{t} x H x\right)-\bar{x}^{t} \bar{x} /\left({ }^{t} \bar{x} H \bar{x}\right),
$$

which must be positive definite. If $x_{4}=0$ then the $(4,4)$ component of $(5)$ is -1 , which implies that (5) can not be positive definite. Thus we have $x_{4} \neq 0$. Put

$$
\eta=\left(\begin{array}{ccc}
x_{4} & 0 & 0 \\
0 & x_{4} & 0 \\
0 & 0 & x_{4} \\
x_{1} & x_{2} & x_{3}
\end{array}\right) ;
$$

note that $(\eta, x) \in G L_{4}(\mathbb{C})$ and that ${ }^{t} x H \eta=(0,0,0)$. We have

$$
{ }^{t} \overline{(\eta, x) H}\left(H-\frac{x^{t} x}{{ }^{t} x H x}-\frac{\bar{x}^{t} \bar{x}}{{ }^{t} \bar{x} H \bar{x}}\right) H(\eta, x)=\left(\begin{array}{cc}
{ }^{t} \bar{\eta} H \eta & 0 \\
{ }^{t} 0 & -{ }^{t} \bar{x} H x
\end{array}\right) .
$$

If

$$
-\bar{x} H x=-\left|x_{1}\right|^{2}-\left|x_{2}\right|^{2}-\left|x_{3}\right|^{2}+\left|x_{4}\right|^{2}>0
$$

then the $3 \times 3$ matrix

$$
{ }^{t} \bar{\eta} H \eta=\left|x_{4}\right|^{2} I_{3}-\left(\begin{array}{c}
\bar{x}_{1} \\
\bar{x}_{2} \\
\bar{x}_{3}
\end{array}\right)\left(x_{1}, x_{2}, x_{3}\right)
$$

is positive definite. Hence the matrix (5) is positive definite if and only if

$$
{ }^{t} \bar{x} H x=\left|x_{1}\right|^{2}+\left|x_{2}\right|^{2}+\left|x_{3}\right|^{2}-\left|x_{4}\right|^{2}<0 .
$$


We embedded the domain $\mathbb{B}^{3}=\left\{\left.x \in \mathbb{P}^{3}\right|^{t} \bar{x} H x<0\right\}$ in $\mathbb{S}^{4}$ by the map

$$
\jmath: \mathbb{B}^{3} \ni x \mapsto \Omega=\omega\left[I_{4}-(1-\omega)\left(x^{t} x H\right) /\left({ }^{t} x H x\right)\right] H \in \mathbb{S}^{4} .
$$

\section{$\S 4$. Monodromy}

Let $\left(\lambda_{1}, \ldots, \lambda_{6}\right)$ vary as an element in $\Lambda$, we have two multi-valued map

$$
\begin{array}{cccc}
\psi: & \Lambda & \rightarrow & \mathbb{B}^{3} \\
\lambda & \mapsto & x=t\left(\int_{A_{1}} \varphi_{1}, \ldots, \int_{A_{4}} \varphi_{1}\right), \\
\tilde{\psi}=\jmath \circ \psi: & \Lambda & \rightarrow & \mathbb{S}^{4} \\
\lambda & \mapsto & \Omega=\jmath(\psi(\lambda)) .
\end{array}
$$

We call them period maps. The map $\psi$ and its monodromy group were studied in [3], [13], [14] and [15], the results are as follows.

Fact 4.1. The image of $\psi$ is open dense in $\mathbb{B}^{3}$. The monodromy group of $\psi$ is conjugate to the congruence subgroup

$$
\Gamma(1-\omega)=\left\{g \in \Gamma \mid g \equiv I_{4} \bmod (1-\omega)\right\}
$$

of the modular group

$$
\Gamma=\left\{g \in G L_{4}(\mathbb{Z}[\omega]) \mid{ }^{t} \bar{g} H g=H\right\} .
$$

The Satake compactification $\hat{\mathbb{B}}^{3} / \Gamma(1-\omega)$ of $\mathbb{B}^{3} / \Gamma(1-\omega)$ is isomorphic to $Y$.

For a column vector $v \in \mathbb{C}^{4}$ such that ${ }^{t} \bar{v} H v \neq 0$, we define reflections $R^{\omega}(v)$ and $R^{\zeta}(v)$ with root $v$ and exponent $\omega$ and $\zeta=-\omega^{2}$, respectively, as

$$
\begin{aligned}
R^{\omega}(v) & =I_{4}-(1-\omega) v\left({ }^{t} \bar{v} H v\right)^{-1}{ }^{t} \bar{v} H, \\
R^{\zeta}(v) & =I_{4}-(1-\zeta) v\left({ }^{t} \bar{v} H v\right)^{-1}{ }^{t} \bar{v} H .
\end{aligned}
$$

It is shown in [2] that $\Gamma(1-\omega)$ can be generated by fifteen reflections $R_{i j}^{\omega}=$ $R^{\omega}\left(v_{i j}\right)(1 \leq i<j \leq 6)$ and that $\Gamma$ by $-I_{4}$ and five reflections $R_{i, i+1}^{\zeta}=$ $R^{\zeta}\left(v_{i, i+1}\right)(1 \leq i \leq 5)$, where

$$
\begin{aligned}
& v_{12}={ }^{t}(1,0,0,0), \quad v_{13}={ }^{t}(-1,1,0,1), \quad v_{14}={ }^{t}\left(-1,-\omega^{2}, 0,1\right), \\
& v_{15}={ }^{t}\left(\omega^{2}, 0,-\omega^{2}, 1\right), v_{16}={ }^{t}\left(\omega^{2}, 0, \omega, 1\right), \quad v_{23}={ }^{t}\left(\omega^{2}, 1,0,1\right), \\
& v_{24}={ }^{t}\left(\omega^{2},-\omega^{2}, 0,1\right), v_{25}={ }^{t}\left(-\omega, 0,-\omega^{2}, 1\right), v_{26}={ }^{t}(-\omega, 0, \omega, 1) \text {, } \\
& v_{34}={ }^{t}(0,1,0,0), \quad v_{35}={ }^{t}(0,-\omega, \omega, 1), \quad v_{36}={ }^{t}(0,-\omega,-1,1), \\
& v_{45}={ }^{t}(0,1, \omega, 1), \quad v_{46}={ }^{t}(0,1,-1,1), \quad v_{56}={ }^{t}(0,0,1,0) \text {. }
\end{aligned}
$$


The reflections correspond to the following movements of $\lambda_{i}$ 's. When $\lambda_{i}$ goes near to $\lambda_{j}$ in the upper half space and turns around $\lambda_{j}$ and returns, $x$ becomes $R_{i j}^{\omega} x$. When $\lambda_{i}$ and $\lambda_{j}$ are exchanged in the upper half space, $x$ becomes $R_{i j}^{\zeta} x$. Since $R_{i, i+1}^{\zeta}$ 's are representations of braids, they satisfy

$$
R_{i-1, i}^{\zeta} R_{i, i+1}^{\zeta} R_{i-1, i}^{\zeta}=R_{i, i+1}^{\zeta} R_{i-1, i}^{\zeta} R_{i, i+1}^{\zeta} \quad(2 \leq i \leq 5) .
$$

The embedding $\jmath$ induces the following homomorphism from $U(3,1 ; \mathbb{C})$ to

$$
\begin{gathered}
S p(8, \mathbb{R})=\left\{g \in G L_{8}(\mathbb{R}) \mid{ }^{t} g J g=J=\left(\begin{array}{cc}
O & -I_{4} \\
I_{4} & O
\end{array}\right)\right\}: \\
\tilde{\jmath}: U(3,1 ; \mathbb{C}) \ni P+\omega Q \mapsto\left(\begin{array}{cc}
P & Q H \\
-H Q & H(P-Q) H
\end{array}\right) \in S p(8, \mathbb{R}),
\end{gathered}
$$

where $P$ and $Q$ are real $4 \times 4$ matrices. Note that

$$
\begin{aligned}
\tilde{\jmath}^{-1}: S p(8, \mathbb{R}) & \supset \tilde{\jmath}(U(3,1 ; \mathbb{C})) \ni\left(\begin{array}{ll}
A & B \\
C & D
\end{array}\right) \\
& \mapsto A+\omega B H=(-H C+H D H)-\omega H C \in U(3,1 ; \mathbb{C}) .
\end{aligned}
$$

Let us express the images of $R^{\omega}(v)$ and $R^{\zeta}(v)$ under the map $\tilde{\jmath}$. The image of $\omega I_{4}$ under $\tilde{\jmath}$ is given by

$$
W=\left(\begin{array}{cc}
O & H \\
-H & -I_{4}
\end{array}\right) \in S p(8, \mathbb{Z}) .
$$

For a column vector $v=a+\omega b\left(a, b \in \mathbb{R}^{4}\right)$, define column vectors $v_{1}=$ $\left(\begin{array}{c}a \\ -H b\end{array}\right)$ and $v_{2}=W v_{1}$ and form a $(8 \times 2)$ matrix $V=\left(v_{1}, v_{2}\right)$. Straightforward calculation shows the following.

Proposition 4.1. If ${ }^{t} \bar{v} H v \neq 0$, then $\tilde{\jmath}\left(R^{\omega}(v)\right)=\tilde{R}^{\omega}(v)$ and $\tilde{\jmath}\left(R^{\zeta}(v)\right)=$ $\tilde{R}^{\zeta}(v)$ are given by

$$
I_{8}-\left(I_{8}-W\right) V\left({ }^{t} V J V\right)^{-1}{ }^{t} V J, \quad I_{8}-\left(I_{8}+W^{2}\right) V\left({ }^{t} V J V\right)^{-1 t} V J,
$$

respectively.

Systems of generators of $\tilde{\Gamma}(1-\omega)=\tilde{\jmath}(\Gamma(1-\omega))$ and $\tilde{\Gamma}=\tilde{\jmath}(\Gamma)$ are given by $\tilde{R}_{i j}^{\omega}$ 's and $\tilde{R}_{i, i+1}^{\zeta}$ 's. 


\section{§5. Riemann Theta Constants}

The Riemann theta function

$$
\vartheta(z, \tau)=\sum_{n=\left(n_{1}, \ldots, n_{r}\right) \in \mathbb{Z}^{r}} \exp \left[\pi \sqrt{-1}\left(n \tau^{t} n+2 n^{t} z\right)\right]
$$

is holomorphic on $\mathbb{C}^{r} \times \mathbb{S}^{r}$ and satisfies

$$
\vartheta(z+p, \tau)=\vartheta(z, \tau), \quad \vartheta(z+p \tau, \tau)=\exp \left[-\pi \sqrt{-1}\left(p \tau^{t} p+2 z^{t} p\right)\right] \vartheta(z, \tau),
$$

where $\mathbb{S}^{r}$ is the Siegel upper half space of degree $r$ and $p \in \mathbb{Z}^{r}$. It is well known that for $(z, \tau) \in \mathbb{C} \times \mathbb{H}, \vartheta(z, \tau)=0$ if and only if $z=(1+\tau) / 2+p+q \tau(p, q \in \mathbb{Z})$.

The theta function $\vartheta_{a, b}(z, \tau)$ with characteristics $a, b$ is defined by

$$
\begin{aligned}
\vartheta_{a, b}(z, \tau) & =\exp \left[\pi \sqrt{-1}\left(a \tau^{t} a+2 a^{t}(z+b)\right)\right] \vartheta(z+a \tau+b, \tau) \\
& =\sum_{n \in \mathbb{Z}^{n}} \exp \left[\pi \sqrt{-1}\left((n+a) \tau^{t}(n+a)+2(n+a)^{t}(z+b)\right)\right],
\end{aligned}
$$

where $a, b \in \mathbb{Q}^{r}$. Note that

$$
\vartheta_{-a,-b}(z, \tau)=\vartheta_{a, b}(-z, \tau), \quad \vartheta_{a+p, b+q}(z, \tau)=\exp \left(2 \pi \sqrt{-1} a^{t} q\right) \vartheta_{a, b}(z, \tau)
$$

The function $\vartheta_{a, b}(\tau)=\vartheta_{a, b}(0, \tau)$ of $\tau$ is called the theta constant with characteristics $a, b$. If $\tau$ is diagonal, then this function becomes the product of Jacobi's theta constants:

$$
\vartheta_{a, b}(\tau)=\prod_{i=1}^{r} \vartheta_{a_{i}, b_{i}}\left(\tau_{i}\right)
$$

where

$$
a=\left(a_{1}, \ldots, a_{r}\right), b=\left(b_{1}, \ldots, b_{r}\right), \tau=\operatorname{diag}\left(\tau_{1}, \ldots, \tau_{r}\right) .
$$

The following transformation formula can be found in [7] p.176.

Fact 5.1. For any $g=\left(\begin{array}{ll}A & B \\ C & D\end{array}\right) \in S p(2 r, \mathbb{Z})$ and $(a, b) \in \mathbb{Q}^{2 r}$, we put

$$
\begin{aligned}
g \cdot(a, b)= & (a, b) g^{-1}+\frac{1}{2}\left(\operatorname{dv}\left(C^{t} D\right), \operatorname{dv}\left(A^{t} B\right)\right) \\
\phi_{(a, b)}(g)= & -\frac{1}{2}\left(a^{t} D B^{t} a-2 a^{t} B C{ }^{t} b+b^{t} C A^{t} b\right) \\
& +\frac{1}{2}\left(a^{t} D-b{ }^{t} C\right){ }^{t}\left(\operatorname{dv}\left(A^{t} B\right)\right),
\end{aligned}
$$


where $\operatorname{dv}(A)$ is the row vector consisting of the diagonal components of $A$. Then for every $g \in S p(2 r, \mathbb{Z})$, we have

$$
\begin{aligned}
\vartheta_{g \cdot(a, b)}((A \tau & \left.+B)(C \tau+D)^{-1}\right) \\
& =\kappa(g) \exp \left(2 \pi \sqrt{-1} \phi_{(a, b)}(g)\right) \operatorname{det}(C \tau+D)^{\frac{1}{2}} \vartheta_{(a, b)}(\tau),
\end{aligned}
$$

in which $\kappa(g)^{2}$ is a 4-th root of 1 depending only on $g$.

Proposition 5.1. There are $81=3^{4}$ theta characteristics

$$
(a, b)=\left(a_{1}, \ldots, a_{4}, b_{1}, \ldots, b_{4}\right)
$$

such that

$$
g \cdot(a, b) \equiv(a, b) \bmod \mathbb{Z}^{8}
$$

for any $g \in \tilde{\Gamma}(1-\omega) \subset S p(8, \mathbb{Z})$; they are given by

$$
b=-a H, a_{i} \in\left\{\frac{1}{6}, \frac{3}{6}, \frac{5}{6}\right\} \quad(i=1, \ldots, 4) .
$$

Proof. Since

$$
W \cdot(a, b)=(-a+b H,-a H)+\frac{1}{2}(1,1,1,-1,0,0,0,0),
$$

we have

$$
-a H \equiv b,-2 a+\frac{1}{2}(1,1,1,-1) \equiv a \bmod \mathbb{Z}^{4} .
$$

Thus we have the condition (8). It is easy to check such theta characteristics are invariant under the action on 15 reflections $\tilde{R}_{i j}^{\omega}$.

We label the 81 characteristics $a$ 's by combinatorics of six letters; they are classified to 4 classes. The list of the correspondence between the label of $a$ and $6 a$ is as follows:

$$
\begin{array}{lll}
(12 ; 34 ; 56) \leftrightarrow \pm(3,3,3,-1) & (12 ; 35 ; 46) \leftrightarrow \pm(3,1,1,-3) & (12 ; 36 ; 45) \leftrightarrow \pm(3,1,-1,-3) \\
(13 ; 24 ; 56) \leftrightarrow \pm(1,1,3,-3) & (13 ; 25 ; 46) \leftrightarrow \pm(1,-1,1,-1) & (13 ; 26 ; 45) \leftrightarrow \pm(-1,1,1,1) \\
(14 ; 23 ; 56) \leftrightarrow \pm(1,-1,3,-3) & (14 ; 25 ; 36) \leftrightarrow \pm(1,1,1,-1) & (14 ; 26 ; 35) \leftrightarrow \pm(1,1,-1,-1) \\
(15 ; 23 ; 46) \leftrightarrow \pm(1,1,-1,1) & (15 ; 24 ; 36) \leftrightarrow \pm(1,-1,-1,1) & (15 ; 26 ; 34) \leftrightarrow \pm(1,3,1,-3) \\
(16 ; 23 ; 45) \leftrightarrow \pm(1,1,1,1) & (16 ; 24 ; 35) \leftrightarrow \pm(1,-1,1,1) & (16 ; 25 ; 34) \leftrightarrow \pm(1,3,-1,-3)
\end{array}
$$




$$
\begin{gathered}
\left(1^{2} 2\right) \leftrightarrow(1,3,3,3) \quad\left(1^{2} 3\right) \leftrightarrow(5,1,3,5) \quad\left(1^{2} 4\right) \leftrightarrow(5,5,3,5) \\
\left(1^{2} 5\right) \leftrightarrow(5,3,1,1) \quad\left(1^{2} 6\right) \leftrightarrow(5,3,5,1) \quad\left(2^{2} 3\right) \leftrightarrow(1,1,3,5) \\
\left(2^{2} 4\right) \leftrightarrow(1,5,3,5) \quad\left(2^{2} 5\right) \leftrightarrow(1,3,1,1) \quad\left(2^{2} 6\right) \leftrightarrow(1,3,5,1) \\
\left(3^{2} 4\right) \leftrightarrow(3,1,3,3) \quad\left(3^{2} 5\right) \leftrightarrow(3,5,1,5) \quad\left(3^{2} 6\right) \leftrightarrow(3,5,5,5) \\
\left(4^{2} 5\right) \leftrightarrow(3,1,1,5) \quad\left(4^{2} 6\right) \leftrightarrow(3,1,5,5) \quad\left(5^{2} 6\right) \leftrightarrow(3,3,1,3) \\
\left(i j^{2}\right) \leftrightarrow-a \text { for }\left(i^{2} j\right) \quad 1 \leq i<j \leq 6, \\
(123) \leftrightarrow(3,1,3,5) \quad(124) \leftrightarrow(3,5,3,5) \quad(125) \leftrightarrow(3,3,1,1) \\
(126) \leftrightarrow(3,3,5,1) \quad(134) \leftrightarrow(1,3,3,1) \quad(135) \leftrightarrow(1,1,1,3) \\
(136) \leftrightarrow(1,1,5,3) \quad(145) \leftrightarrow(1,5,1,3) \quad\left\{\begin{array}{c}
(146) \\
(156) \leftrightarrow(1,3,3,5) \quad(l m n) \leftrightarrow-a \text { for }(i j k) \quad\{i, j, k, l, m, n\}=\{1, \ldots, 6\}
\end{array}\right. \\
\quad(123456) \leftrightarrow(3,3,3,3) .
\end{gathered}
$$

The first class is characterized by $(6 a) H^{t}(6 a) \equiv 2 \bmod 24$ and the characteristics $(a,-a H)$ with label $(i j ; k l ; m n)$ is invariant under the actions $\tilde{R}_{i j}^{\zeta}$, $\tilde{R}_{k l}^{\zeta}$ and $\tilde{R}_{m n}^{\zeta}$; the second class is characterized by $(6 a) H^{t}(6 a) \equiv 10 \bmod 24$ and the characteristics $(a,-a H)$ with label $\left(i^{2} j\right)$ is invariant under the actions $\tilde{R}_{k l}^{\zeta}(\{i, j\} \cap\{k, l\}=\emptyset)$ and $\tilde{R}_{i j}^{\zeta} \cdot(a,-a H)$ is $(-a, a H)$ with label $\left(i j^{2}\right)$; the third class is characterized by $(6 a) H^{t}(6 a) \equiv 18 \bmod 24$ and the characteristics $(a,-a H)$ with label $(i j k)$ is invariant under the actions $\tilde{R}_{l m}^{\zeta}(\{i, j, k\} \cap\{l, m\}=$ $\emptyset$ or $\{l, m\})$.

We denote $\vartheta_{a,-a H}(\Omega)$ by $\vartheta_{[6 a]}(\Omega)$ or $\vartheta(i j ; k l ; m n), \vartheta\left(i^{2} j\right), \vartheta(i j k)$ and $\vartheta(123456)$ for corresponding characteristics $a$. Note that for $p, q \in \mathbb{Z}^{4}$,

$$
\begin{aligned}
\vartheta(a(\Omega & -H)+p \Omega+q, \Omega) \\
& =\exp \left[-\pi \sqrt{-1}\left(p \Omega{ }^{t} p+2 p(\Omega-H){ }^{t} a\right)\right] \vartheta(a(\Omega-H), \Omega) \\
& =\exp \left[-\pi \sqrt{-1}\left(p \Omega{ }^{t} p+2 p(\Omega-H){ }^{t} a+a \Omega^{t} a-2 a H^{t} a\right)\right] \vartheta_{a,-a H}(\Omega) \\
& =\exp \left[2 \pi \sqrt{-1}(a+p) H^{t}(a+p)\right] \exp \left[-\pi \sqrt{-1}(a+p) \Omega^{t}(a+p)\right] \vartheta_{[a]}(\Omega) .
\end{aligned}
$$


Proposition 5.2. The theta constants $\vartheta\left(i^{2} j\right), \vartheta(i j k)$ and $\vartheta(123456)$ are identically zero on $\jmath\left(\mathbb{B}^{3}\right)$. The theta constants $\vartheta(i j ; k l ; m n)$ are not identically zero on $\jmath\left(\mathbb{B}^{3}\right)$.

Proof. We apply Fact 5.1 for

$$
\tau=\Omega=\jmath(x), \quad g=W=\left(\begin{array}{cc}
0 & H \\
-H & -I_{4}
\end{array}\right), \quad(a, b)=(a,-a H) .
$$

Note that

$$
W \cdot \Omega=\Omega, \quad W \cdot(a,-a H)=\left(a-\left(3 a-\frac{1}{2} \operatorname{diag}(H)\right),-a H\right)
$$

and that

$$
\phi_{(a,-a H)}(W)=\frac{3}{2} a H^{t} a=\frac{1}{24}(6 a) H^{t}(6 a), \quad \operatorname{det}(C \Omega+D)=\omega .
$$

Since $\kappa(W)$ is an 8 -th root of 1 , the sufficient condition for

$$
\kappa(W) \exp \left(2 \pi \sqrt{-1} \phi_{(a, b)}(W)\right) \operatorname{det}(C \Omega+D)^{\frac{1}{2}}=1
$$

is $(6 a) H^{t}(6 a) \equiv 2 \bmod 24$. If $(6 a) H^{t}(6 a) \not \equiv 2 \bmod 24$, then $\vartheta_{a,-a H}(\Omega)$ vanishes. Thus the theta constants $\vartheta\left(i^{2} j\right), \vartheta(i j k)$ and $\vartheta(123456)$ are identically zero on $\jmath\left(\mathbb{B}^{3}\right)$.

For $a=\left(\frac{1}{6}, \ldots, \frac{1}{6}\right)$ and $x=(0,0,0,1), \vartheta_{a,-a H}(\Omega)$ reduces to

$$
\vartheta_{\left(\frac{1}{6}, \frac{-1}{6}\right)}(\omega)^{3} \vartheta_{\left(\frac{1}{6}, \frac{1}{6}\right)}\left(-\omega^{2}\right),
$$

which does not vanish. Hence $\vartheta(i j ; k l ; m n)$ 's survive. Note that $\kappa(W)^{2}=-1$ by $(9)$.

Proposition 5.3. We have

$$
\begin{aligned}
& \vartheta(i, i+1 ; k l ; m n)\left(\tilde{R}_{i, i+1}^{\zeta} \cdot \jmath(x)\right)^{3}=-\chi\left(\tilde{R}_{i, i+1}^{\zeta}\right) \vartheta(i, i+1 ; k l ; m n)(\jmath(x))^{3}, \\
& \vartheta(i k ; i+1, l ; m n)\left(\tilde{R}_{i, i+1}^{\zeta} \cdot \jmath(x)\right)^{3}=\chi\left(\tilde{R}_{i, i+1}^{\zeta}\right) \vartheta(i l ; i+1, k ; m n)(\jmath(x))^{3},
\end{aligned}
$$

where

$$
\chi\left(\tilde{R}_{i, i+1}^{\zeta}\right)=\left(\frac{{ }^{t}\left(R_{i, i+1}^{\zeta} x\right) H\left(R_{i, i+1}^{\zeta} x\right)}{{ }^{t} x H x}\right)^{3 / 2},
$$

which takes 1 on the mirror of $R_{i, i+1}^{\zeta}$. 
Proof. $\quad$ For $\tilde{R}_{i, i+1}^{\zeta}=\left(\begin{array}{cc}A & B \\ C & D\end{array}\right)$, straightforward calculation shows

$$
\operatorname{det}(C \jmath(x)+D)=\frac{{ }^{t}\left(R_{i, i+1}^{\zeta} x\right) H\left(R_{i, i+1}^{\zeta} x\right)}{\operatorname{det}\left(R_{i, i+1}^{\zeta}{ }^{t} x H x\right.}=\frac{{ }^{t}\left(R_{i, i+1}^{\zeta} x\right) H\left(R_{i, i+1}^{\zeta} x\right)}{-\omega^{2}{ }^{t} x H x} .
$$

By computing $\phi_{a, b}\left(\tilde{R}_{i, i+1}^{\zeta}\right)$ in Fact 5.1 and using $(7)$, we have

$$
\begin{aligned}
& \vartheta(i, i+1 ; k l ; m n)\left(\tilde{R}_{i, i+1}^{\zeta} \cdot \jmath(x)\right)^{3}=-c \chi\left(\tilde{R}_{i, i+1}^{\zeta}\right) \vartheta(i, i+1 ; k l ; m n)(\jmath(x))^{3}, \\
& \vartheta(i k ; i+1, l ; m n)\left(\tilde{R}_{i, i+1}^{\omega} \cdot \jmath(x)\right)^{3}=c \chi\left(\tilde{R}_{i, i+1}^{\zeta}\right) \vartheta(i l ; i+1, k ; m n)(\jmath(x))^{3},
\end{aligned}
$$

where $c$ is a certain constant depending only on $\tilde{R}_{i, i+1}^{\zeta}$. If we restrict $\jmath(x)$ on the mirror of $R_{i, i+1}^{\zeta}$, we have

$$
\tilde{R}_{i, i+1}^{\zeta} \cdot \jmath(x)=\jmath(x), \quad \chi\left(\tilde{R}_{i, i+1}^{\zeta}\right)=\left(\frac{{ }^{t}\left(R_{i, i+1}^{\zeta} x\right) H\left(R_{i, i+1}^{\zeta} x\right)}{{ }^{t} x H x}\right)^{3 / 2}=1 .
$$

Since $\vartheta(i, k ; i+1, l ; m n)=\vartheta(i, l ; i+1, k ; m n)$ on the mirror of $R_{i, i+1}^{\zeta}$ and it does not vanish, the constant $c$ must be 1 .

Since $\tilde{R}_{p q}^{\zeta}$ can be expressed in terms of $\tilde{R}_{i, i+1}^{\zeta}$ and $\tilde{R}_{p q}^{\omega}=\left(\tilde{R}_{p q}^{\zeta}\right)^{2}$, we have the following two propositions.

\section{Proposition 5.4. We have}

$$
\vartheta(i j ; k l ; m n)\left(\tilde{R}_{p q}^{\omega} \cdot \jmath(x)\right)^{3}=\chi\left(\tilde{R}_{p q}^{\omega}\right) \vartheta(i j ; k l ; m n)(\jmath(x))^{3},
$$

where

$$
\chi\left(\tilde{R}_{p q}^{\omega}\right)=\left(\frac{{ }^{t}\left(R_{p q}^{\omega} x\right) H\left(R_{p q}^{\omega} x\right)}{{ }^{t} x H x}\right)^{3 / 2},
$$

which takes 1 on the mirror of $R_{p q}^{\omega}$.

Proposition 5.5. The function $\vartheta(i j ; k l ; m n)(\jmath(x))$ vanishes on the $\Gamma(1-\omega)$ orbits of the mirrors of $R_{i j}^{\omega}, R_{k l}^{\omega}$ and $R_{m n}^{\omega}$.

Proof. By Proposition 5.3, when we restrict $\jmath(x)$ on the mirrors of $R_{12}^{\omega}$, $R_{34}^{\omega}$ and $R_{56}^{\omega}$, we have

$$
\vartheta(12 ; 34 ; 56)(\jmath(x))^{3}=-\vartheta(12 ; 34 ; 56)(\jmath(x))^{3}=0 .
$$

For the $\Gamma(1-\omega)$ orbits, use the previous proposition. In oder to show for general $\vartheta(i j ; k l ; m n)(\jmath(x))$ 's, use Proposition 5.3. 


\section{$\S 6 . \quad$ The Inverse of the Period Map}

Proposition 6.1. $\quad$ Let $\Omega$ be the period matrix of

$$
C(\lambda): w^{3}=z(z-1)\left(z-\ell_{1}\right)\left(z-\ell_{2}\right)\left(z-\ell_{3}\right)
$$

given in Proposition 3.1. We have

$$
\begin{aligned}
& \ell_{1}=\frac{\vartheta^{3}(13 ; 24 ; 56)(\Omega)}{\vartheta^{3}(14 ; 23 ; 56)(\Omega)}, \\
& \ell_{2}=\frac{\vartheta^{3}(13 ; 25 ; 46)(\Omega)}{\vartheta^{3}(15 ; 23 ; 46)(\Omega)}, \\
& \ell_{3}=\frac{\vartheta^{3}(13 ; 26 ; 45)(\Omega)}{\vartheta^{3}(16 ; 23 ; 45)(\Omega)} .
\end{aligned}
$$

Proposition 6.2. $\quad$ For the period matrix $\Omega$ of $C(\lambda)$, linear and cubic relations among $\vartheta^{3}(i j ; k l ; m n)(\Omega)$ coincide with the defining equations of $Y \subset$ $\mathbb{P}^{14}$ :

$$
\begin{aligned}
& \vartheta^{3}(i j ; k l ; m n)(\Omega)-\vartheta^{3}(i k ; j l ; m n)(\Omega)+\vartheta^{3}(i l ; j k ; m n)(\Omega)=0, \\
& \vartheta^{3}(i j ; k l ; m n)(\Omega) \vartheta^{3}(i k ; j n ; l m)(\Omega) \vartheta^{3}(i m ; j l ; k n)(\Omega) \\
& \quad=\vartheta^{3}(i j ; k n ; l m)(\Omega) \vartheta^{3}(i k ; j l ; m n)(\Omega) \vartheta^{3}(i m ; j n ; k l)(\Omega) .
\end{aligned}
$$

Propositions 6.1 and 6.2 imply the following.

Theorem 6.1. $\quad$ Let $\Theta$ be the map from $\mathbb{B}^{3} / \Gamma(1-\omega)$ to $Y$ defined by

$$
x \mapsto\left[\ldots, y_{\langle i j ; k l ; m n\rangle}, \ldots\right]=\left[\ldots, \vartheta^{3}(i j ; k l ; m n)(\jmath(x)), \ldots\right] .
$$

We have the following $S_{6}$-equivariant commutative diagram:

$$
\begin{array}{ll}
\Lambda & \stackrel{\psi}{\longrightarrow} \mathbb{B}^{3} / \Gamma(1-\omega) \\
\iota \downarrow & \Theta \swarrow \\
Y \subset \mathbb{P}^{14} .
\end{array}
$$

In order to prove Propositions 6.1 and 6.2, we state two facts in [8]; the one is Riemann's theorem and the other is Abel's theorem.

Fact 6.1. We suppose $z$ is a fix point on the Jacobi variety $J a c(R)$ of a Riemann surface $R$ of genus $r$. The multi-valued function $\vartheta\left(z+\int_{P_{0}}^{P} \varphi, \tau\right)$ of 
$P$ on $X$ has $r$ zeros $P_{1}, \ldots, P_{r}$ provided not to be constantly zero, where $\varphi=$ $\left(\varphi_{1}, \ldots, \varphi_{r}\right)$ is the normalized basis of the vector space of holomorphic 1-forms on $R$ such that $\left(\int_{B_{i}} \varphi_{j}\right)_{i j}=I_{r}$ for a symplectic basis $\left\{A_{1}, \ldots, A_{r}, B_{1}, \ldots, B_{r}\right\}$ of $H_{1}(R, \mathbb{Z})$, and $\tau=\left(\int_{A_{i}} \varphi_{j}\right)_{i j}$. Moreover, there exists a point $\Delta$ on $\operatorname{Jac}(R)$ called Riemann's constant such that

$$
z=\Delta-\sum_{i=1}^{r} \int_{P_{0}}^{P_{i}} \varphi .
$$

Fact 6.2. $\quad$ Let $R$ be a Riemann surface of genus $r$ with an initial point $P_{0}$. Suppose $\sum_{i=1}^{d} P_{i}$ and $\sum_{i=1}^{d} Q_{i}$ be effective divisors of degree d satisfying

$$
\sum_{i=1}^{d} \int_{P_{0}}^{P_{i}} \varphi=\sum_{i=1}^{d} \int_{P_{0}}^{Q_{i}} \varphi
$$

where $\varphi$ is the normalized basis of vector space of holomorphic 1-forms on $R$. Then there exists a meromorphic function $f$ on $R$ such that

$$
(f)=\sum_{i=1}^{d} Q_{i}-\sum_{i=1}^{d} P_{i}
$$

$f$ can be expressed as

$$
f(P)=c \frac{\prod_{i=1}^{d} \vartheta\left(e+\int_{Q_{i}}^{P} \varphi, \tau\right)}{\prod_{i=1}^{d} \vartheta\left(e+\int_{P_{i}}^{P} \varphi, \tau\right)},
$$

where $c$ is a constant, $\tau$ is the period matrix of $R$, e satisfies $\vartheta(e)=0$,

$$
\vartheta\left(e+\int_{P_{i}}^{P} \varphi, \tau\right) \not \equiv 0, \quad \vartheta\left(e+\int_{Q_{i}}^{P} \varphi, \tau\right) \not \equiv 0,
$$

as multi-valued functions of $P$ on $R$, and paths from $P_{i}$ and $Q_{i}$ to $P$ are the inverse of the paths in (15) followed by a common path from $P_{0}$ to $P$.

Proof of Proposition 6.1. We take $R$ as

$$
C: w^{3}=z(z-1)\left(z-\ell_{1}\right)\left(z-\ell_{2}\right)\left(z-\ell_{3}\right)
$$

with the initial point $P_{0}=(0,0)$ and put

$$
P_{\infty}=(\infty, \infty), \quad P_{1}=(1,0), \quad P_{\ell_{i}}=\left(\ell_{i}, 0\right)(i=1,2,3) .
$$


Let us define a meromorphic function $f$ on $C$ by $C \ni(z, w) \mapsto z$, then

$$
(f)=3 P_{0}-3 P_{\infty}
$$

We construct a meromorphic function on $C$ with poles $3 P_{\infty}$ and zeros $3 P_{0}$ by following the recipe given in Fact 6.2 . Let $\gamma_{i}\left(z_{1}, z_{2}\right)(i=1,2,3)$ be a path in $C$ from $\left(z_{1}, w_{1}\right)$ to $\left(z_{2}, w_{2}\right)$ in the $i$-th sheet. Since $\omega^{2}+\omega+1=0$, we have

$$
\sum_{i=1}^{3} \int_{\gamma_{i}(0, \infty)} \varphi=(0,0,0,0)
$$

for three paths $\gamma_{i}(0, \infty)$ from $P_{0}$ to $P_{\infty}$. We give the following table:

$$
\begin{aligned}
\int_{\gamma_{1}(\infty, 0)} \varphi & =\frac{1}{3} \int_{A_{1}-B_{1}} \varphi, \quad \int_{\gamma_{2}(\infty, 0)} \varphi=\frac{1}{3} \int_{-2 A_{1}-B_{1}} \varphi \\
\int_{\gamma_{3}(\infty, 0)} \varphi & =\frac{1}{3} \int_{A_{1}+2 B_{1}} \varphi, \quad \int_{\gamma_{1}(0,1)} \varphi=\frac{1}{3} \int_{-2 A_{1}+A_{2}-A_{4}-B_{1}+2 B_{2}+2 B_{4}} \varphi \\
\int_{\gamma_{2}(0,1)} \varphi & =\frac{1}{3} \int_{A_{1}+A_{2}-A_{4}+2 B_{1}-B_{2}-B_{4}} \varphi \\
\int_{\gamma_{3}(0,1)} \varphi & =\frac{1}{3} \int_{A_{1}-2 A_{2}+2 A_{4}-B_{1}-B_{2}-B_{4}} \varphi, \quad \int_{\gamma_{1}\left(1, \ell_{1}\right)} \varphi=\frac{1}{3} \int_{A_{2}-B_{2}} \varphi \\
\int_{\gamma_{1}\left(\ell_{1}, \ell_{2}\right)} \varphi & =\frac{1}{3} \int_{-2 A_{2}+A_{3}+2 A_{4}-B_{2}+2 B_{3}-B_{4}} \varphi, \int_{\gamma_{1}\left(\ell_{2}, \ell_{3}\right)} \varphi=\frac{1}{3} \int_{A_{3}-B_{3}} \varphi .
\end{aligned}
$$

Put

$$
e=\frac{1}{6} \int_{3 A_{1}+A_{2}+3 A_{3}+5 A_{4}-3 B_{1}-B_{2}-3 B_{3}+5 B_{4}} \varphi,
$$

corresponding to the characteristic $(3,1,3,5) / 6$ with label (123), and define a meromorphic function $F$ of $P=(z, w)$ on $C$ as

$$
F(P)=\frac{\vartheta\left(e+\int_{\gamma_{1}(0, z)} \varphi, \Omega\right)^{3}}{\prod_{i=1}^{3} \vartheta\left(e+\int_{\gamma_{i}(\infty, 0)+\gamma_{1}(0, z)} \varphi, \Omega\right)},
$$

where $\Omega$ is the period matrix of $C$. Since $\vartheta(123)$ vanishes, we have $\vartheta(e)=0$. We check that neither the denominator nor the numerator of $F$ identically vanishes. We put $P=P_{\ell_{1}}, P_{\ell_{2}}, P_{\ell_{3}}$ and use (6) and (7), then we have

$$
\begin{aligned}
& F\left(P_{\ell_{1}}\right)=c f\left(P_{\ell_{1}}\right)=c \ell_{1}=\exp \left[\frac{\pi \sqrt{-1}}{3}\left(2 \Omega_{11}+1\right)\right] \frac{\vartheta_{[-1,-1,3,-3]}^{3}(\Omega)}{\vartheta_{[1,-1,3,-3]}^{3}(\Omega)}, \\
& F\left(P_{\ell_{2}}\right)=c f\left(P_{\ell_{2}}\right)=c \ell_{2}=\exp \left[\frac{\pi \sqrt{-1}}{3}\left(2 \Omega_{11}+1\right)\right] \frac{\vartheta_{[-1,1,-1,1]}^{3}(\Omega)}{\vartheta_{[1,1,-1,1]}^{3}(\Omega)},
\end{aligned}
$$




$$
F\left(P_{\ell_{3}}\right)=c f\left(P_{\ell_{3}}\right)=c \ell_{3}=\exp \left[\frac{\pi \sqrt{-1}}{3}\left(2 \Omega_{11}+1\right)\right] \frac{\vartheta_{[-1,1,1,1]}^{3}(\Omega)}{\vartheta_{[1,1,1,1]}^{3}(\Omega)},
$$

where $c$ is a constant depending on $\Omega$. By Proposition 5.2, neither the denominator nor the numerator of $F$ identically vanishes.

We put $P=P_{\infty}, P_{0}, P_{1}$; the denominator and the numerator of $F$ vanish at these points by Proposition 5.2. Since $(F)=3 P_{0}-3 P_{\infty}, P_{\infty}$ and $P_{0}$ are zeros of higher order of the denominator and numerator of $F$, respectively. The number of zeros of the denominator and numerator of $F$ are 4 by Fact 6.1 , thus $P_{1}$ is a simple zero. We consider $\lim _{P \rightarrow P_{1}} F(P)$. Let $t$ be a local coordinate for $P$ around $P_{1}$ and $z(t)$ be $\int_{P_{1}}^{P} \varphi$. We have

$$
F(P)=\exp \left[\frac{\pi \sqrt{-1}}{3}\left(2 \Omega_{11}-2\right)\right] \frac{\vartheta_{[-1,-3,-3,-3]}^{3}(z(t), \Omega)}{\vartheta_{[1,3,3,3]}^{3}(z(t), \Omega)} .
$$

When $P \rightarrow P_{1}$, we have $t \rightarrow 0$ and $z(t) \rightarrow(0,0,0,0)$. Since $t=0$ is simple zero, we have

$$
\lim _{t \rightarrow 0} \frac{\vartheta_{[-1,-3,-3,-3]}^{3}(z(t), \Omega)}{\vartheta_{[1,3,3,3]}^{3}(z(t), \Omega)}=\lim _{t \rightarrow 0} \frac{\vartheta_{[1,3,3,3]}^{3}(-z(t), \Omega)}{\vartheta_{[1,3,3,3]}^{3}(z(t), \Omega)}=-1,
$$

which implies $c=\exp \left[(\pi \sqrt{-1} / 3)\left(2 \Omega_{11}+1\right)\right]$. Hence we have the expressions (10), (11) and (12).

Proof of Proposition 6.2. In order to obtain a cubic relation among $\vartheta^{3}$ $(i j ; k l ; m n)$ 's, put

$$
e=\frac{1}{6} \int_{3 A_{1}+5 A_{2}+3 A_{3}+5 A_{4}-3 B_{1}-5 B_{2}-3 B_{3}+5 B_{4}} \varphi
$$

corresponding to the characteristic $(3,5,3,5) / 6$ with label $(124)$, then $\vartheta(e)=0$; and define a meromorphic function $F$ by $(16)$. We have

$$
\begin{gathered}
F\left(P_{1}\right)=c f\left(P_{1}\right)=c=\exp \left[\frac{\pi \sqrt{-1}}{3}\left(2 \Omega_{11}+1\right)\right] \frac{\vartheta_{[-1,1,3,-3]}^{3}(\Omega)}{\vartheta_{[1,1,3,-3]}(\Omega)}, \\
F\left(P_{\ell_{2}}\right)=c f\left(P_{\ell_{2}}\right)=c \ell_{2}=\exp \left[\frac{\pi \sqrt{-1}}{3}\left(2 \Omega_{11}+1\right)\right] \frac{\vartheta_{[-1,-1,-1,1]}^{3}(\Omega)}{\vartheta_{[1,-1,-1,1]}^{3}(\Omega)} \\
F\left(P_{\ell_{3}}\right)=c f\left(P_{\ell_{3}}\right)=c \ell_{3}=\exp \left[\frac{\pi \sqrt{-1}}{3}\left(2 \Omega_{11}+1\right)\right] \frac{\vartheta_{[-1,-1,1,1]}^{3}(\Omega)}{\vartheta_{[1,-1,1,1]}^{3}(\Omega)},
\end{gathered}
$$


and

$$
\begin{aligned}
c \ell_{1} & =c f\left(P_{\ell_{1}}\right)=\lim _{P \rightarrow P_{\ell_{1}}} F(P) \\
& =\exp \left[\frac{\pi \sqrt{-1}}{3}\left(2 \Omega_{11}-2\right)\right] \frac{\vartheta_{[-1,-3,-3,-3]}^{3}\left(\int_{P_{\ell_{1}}}^{P} \varphi, \Omega\right)}{\vartheta_{[1,3,3,3]}^{3}\left(\int_{P_{\ell_{1}}}^{P} \varphi, \Omega\right)} \\
& =\exp \left[\frac{\pi \sqrt{-1}}{3}\left(2 \Omega_{11}+1\right)\right] .
\end{aligned}
$$

These imply

$$
\begin{aligned}
& \ell_{1}=\frac{c f\left(P_{\ell_{1}}\right)}{c f\left(P_{1}\right)}=\frac{\vartheta^{3}(13 ; 24 ; 56)(\Omega)}{\vartheta^{3}(14 ; 23 ; 56)(\Omega)}, \\
& \ell_{2}=\frac{c f\left(P_{\ell_{2}}\right)}{c f\left(P_{1}\right)}=\frac{\vartheta^{3}(14 ; 25 ; 36)(\Omega) \vartheta^{3}(13 ; 24 ; 56)(\Omega)}{\vartheta^{3}(15 ; 24 ; 36)(\Omega) \vartheta^{3}(14 ; 23 ; 56)(\Omega)}, \\
& \ell_{3}=\frac{c f\left(P_{\ell_{3}}\right)}{c f\left(P_{1}\right)}=\frac{\vartheta^{3}(14 ; 26 ; 35)(\Omega) \vartheta^{3}(13 ; 24 ; 56)(\Omega)}{\vartheta^{3}(16 ; 24 ; 35)(\Omega) \vartheta^{3}(14 ; 23 ; 56)(\Omega)} .
\end{aligned}
$$

Compare with the above expression of $\ell_{2}$ and (11), we have a cubic relation among the $\vartheta^{3}(i j ; k l ; m n)$ 's. By letting $S_{6} \simeq \tilde{\Gamma} /\left\langle\tilde{\Gamma}(1-\omega),-I_{4}\right\rangle$ act on theta constants, we have more cubic relations among $\vartheta^{3}(i j ; k l ; m n)$ 's.

Let us lead a linear relation among the $\vartheta^{3}(i j ; k l ; m n)$ 's. We start with the meromorphic function $f^{\prime}:(z, w) \mapsto z-1$; note that $\left(f^{\prime}\right)=3 P_{1}-3 P_{\infty}$. Put

$$
e=\frac{1}{6} \int_{3 A_{1}+A_{2}+3 A_{3}+5 A_{4}-3 B_{1}-B_{2}-3 B_{3}+5 B_{4}} \varphi,
$$

corresponding to the characteristic $(3,1,3,5) / 6$ with label $(123)$, and define a meromorphic function $F^{\prime}$ of $P=(z, w)$ on $C$ as

$$
F^{\prime}(P)=\frac{\prod_{i=1}^{3} \vartheta\left(e+\int_{\gamma_{i}(1,0)+\gamma_{1}(0, z)} \varphi, \Omega\right)}{\prod_{i=1}^{3} \vartheta\left(e+\int_{\gamma_{i}(\infty, 0)+\gamma_{1}(0, z)} \varphi, \Omega\right)} .
$$

Since $\vartheta(123)$ vanishes, we have $\vartheta(e)=0$. We consider $\lim _{P \rightarrow P_{0}} F^{\prime}(P)$ and put $P=P_{\ell_{1}}$ then we have

$$
\begin{aligned}
& F^{\prime}\left(P_{0}\right)=c f^{\prime}\left(P_{0}\right)=-c=\lim _{P \rightarrow P_{0}} K \exp \left[\frac{4 \pi \sqrt{-1}}{3}\right] \frac{\vartheta_{[-5,-1,-3,-5]}^{3}\left(\int_{P_{0}}^{P} \varphi, \Omega\right)}{\vartheta_{[5,1,3,5]}^{3}\left(\int_{P_{0}}^{P} \varphi, \Omega\right)} \\
& F^{\prime}\left(P_{\ell_{1}}\right)=c f^{\prime}\left(P_{\ell_{1}}\right)=c\left(\ell_{1}-1\right)=K \exp \left[\frac{4 \pi \sqrt{-1}}{3}\right] \frac{\vartheta_{[3,3,3,-1]}^{3}(\Omega)}{\vartheta_{[1,-1,3,-3)}^{3}(\Omega)},
\end{aligned}
$$


where

$$
K=\exp \left[-\frac{2 \pi \sqrt{-1}}{3} e^{\prime} \Omega^{t}\left(e^{\prime}-e_{1}\right)+\frac{4 \pi \sqrt{-1}}{3} e^{\prime} H^{t}\left(e^{\prime}-e_{1}\right)\right]
$$

and $e^{\prime}=(1,-1,0,1)$. Now we have the expression

$$
\ell_{1}-1=\frac{\vartheta^{3}(12 ; 34 ; 56)(\Omega)}{\vartheta^{3}(14 ; 23 ; 56)(\Omega)}
$$

Since we had in (10)

$$
\ell_{1}=\frac{\vartheta^{3}(13 ; 24 ; 56)(\Omega)}{\vartheta^{3}(14 ; 23 ; 56)(\Omega)}
$$

we get a relation

$$
\frac{\vartheta^{3}(12 ; 34 ; 56)(\Omega)}{\vartheta^{3}(14 ; 23 ; 56)(\Omega)}-\frac{\vartheta^{3}(13 ; 24 ; 56)(\Omega)}{\vartheta^{3}(14 ; 23 ; 56)(\Omega)}+1=0,
$$

which is equivalent to

$$
\vartheta^{3}(12 ; 34 ; 56)(\Omega)-\vartheta^{3}(13 ; 24 ; 56)(\Omega)+\vartheta^{3}(14 ; 23 ; 56)(\Omega)=0 .
$$

Action of $S_{6} \simeq \tilde{\Gamma} /\left\langle\tilde{\Gamma}(1-\omega),-I_{4}\right\rangle$ produces the other linear relations among the $\vartheta^{3}(i j ; k l ; m n)$ 's.

\section{$\S 7 . \quad$ Appendix}

In this section, we give a geometrical meaning of the label of $a$ 's. In order to do this, we determine Riemann's constant $\Delta$.

Fact 7.1. Riemann's constant $\Delta$ is given by

$$
\Delta=\sum_{i=1}^{m+r-1} \int_{P_{0}}^{P_{i}} \varphi-\sum_{j=1}^{m} \int_{P_{0}}^{Q_{j}} \varphi
$$

for a certain divisor $D_{0}=\sum_{i=1}^{m+r-1} P_{i}-\sum_{j=1}^{m} Q_{j}$ such that $2 D_{0}$ is linearly equivalent to the canonical divisor of $R$. It is easy to see that Riemann's constant $\Delta$ is a half period on $J a c(R)$ if and only if $(2 r-2) P_{0}$ is a canonical divisor.

For our case, Riemann's constant $\Delta$ is a half period on $\operatorname{Jac}(C(\lambda))$ since we have $6 P_{0}=\left(\varphi_{4}\right)$ for any $C(\lambda)$. 
Proposition 7.1. Riemann's constant $\Delta$ is invariant under the action of the monodromy group $\tilde{\Gamma}(1-\omega)$. Hence we have

$$
\Delta=\left(\frac{1}{2}, \ldots, \frac{1}{2}\right)
$$

Proof. Let $\gamma$ be a closed path in $\Lambda$ and $g \in \tilde{\Gamma}(1-\omega)$ be its representation. Since $\Delta$ is a half period point of $\operatorname{Jac}(C(\lambda))$, it is expressed by $c=\left(c_{1}, \ldots, c_{8}\right)$ $\left(c_{i} \in\{0,1 / 2\}\right)$. When $\lambda$ moves a little, this vector is invariant and presents $\Delta$. By the continuation along $\gamma, \Delta$ is presented by the vector $c$ with respect to the transformed homology basis by $g$; i.e., it is presented by $g \cdot c$ with respect to the initial homology basis.

On the other hand, $\Delta$ is invariant as a point of $\operatorname{Jac}(C(\lambda))$ under the continuation along $\gamma$ with respect to the initial basis by the expression (17). Thus we have $g \cdot c=c$. There is only one half characteristic $(1 / 2, \ldots, 1 / 2)$ invariant under $\tilde{\Gamma}(1-\omega)$.

By straightforward calculation, we have the following proposition giving a geometrical meaning of the label of $a$ 's.

Proposition 7.2. $\quad$ The points $(a,-a H)$ of $J a c(C)$ for a with label $(i j k)$ and $\left(i^{2} j\right)$ are expressed as

$$
\begin{aligned}
& \Delta-\int_{P_{0}}^{P_{\lambda_{i}}} \varphi-\int_{P_{0}}^{P_{\lambda_{j}}} \varphi-\int_{P_{0}}^{P_{\lambda_{k}}} \varphi, \\
& \Delta-2 \int_{P_{0}}^{P_{\lambda_{i}}} \varphi-\int_{P_{0}}^{P_{\lambda_{j}}} \varphi
\end{aligned}
$$

respectively.

We have the necessary and sufficient condition for $\vartheta(z, \tau)=0$.

Fact 7.2. $\quad$ For a period matrix $\tau$ of Riemann's surface $R$ of genus $r$, $\vartheta(z, \tau)=0$ if and only if there exists an effective divisor $\sum_{i=1}^{r-1} P_{i}$ such that

$$
z=\Delta-\sum_{i=1}^{r-1} \int_{P_{0}}^{P_{i}} \varphi .
$$

Proposition 7.3. The theta constant $\vartheta(i j ; k l ; m n)(\jmath(x))$ vanishes only on the $\Gamma(1-\omega)$ orbit of the mirrors of $R_{i j}^{\omega}, R_{k l}^{\omega}$ and $R_{m n}^{\omega}$. 
Proof. The function $\vartheta(13 ; 24 ; 56)(\jmath(x))$ is a non-zero constant times

$$
\vartheta\left(\Delta-\int_{P_{0}}^{P_{\infty}} \varphi-\int_{P_{0}}^{P_{0}} \varphi-\int_{P_{0}}^{P_{1}} \varphi+\int_{P_{0}}^{P_{\ell_{1}}} \varphi, \jmath(x)\right) .
$$

By the previous fact, $\vartheta(13 ; 24 ; 56)(\jmath(x))=0$ if and only if there exists an effective divisor $Q_{1}+Q_{2}+Q_{3}$ such that

$$
Q_{1}+Q_{2}+Q_{3} \equiv P_{\infty}+2 P_{0}+P_{1}-P_{\ell_{1}}=E .
$$

By the Riemann-Roch theorem, the dimension of vector space of meromorphic functions $f$ such that $(f)+E \geq 0$ is equal to that of meromorphic 1-forms $\phi$ such that

$$
(\phi)-E \geq 0
$$

Since we have

$$
\begin{gathered}
\left(\varphi_{1}\right)=P_{\infty}+P_{0}+P_{1}+P_{\ell_{1}}+P_{\ell_{2}}+P_{\ell_{3}}, \quad\left(\varphi_{2}\right)=6 P_{\infty}, \\
\left(\varphi_{3}\right)=3 P_{\infty}+3 P_{0}, \quad\left(\varphi_{4}\right)=6 P_{0},
\end{gathered}
$$

there does not exist a meromorphic 1-from satisfying (18). Thus if $\lambda \in \Lambda$ then no effective divisor $Q_{1}+Q_{2}+Q_{3}$ such that $Q_{1}+Q_{2}+Q_{3} \equiv E$.

The zeros of theta constants on mirrors are studied in [12], which yields this proposition.

\section{Acknowledgements}

The author is grateful to Professors H. Shiga and M. Yoshida for their valuable discussions.

\section{References}

[1] Allcock, D., Carlson, J.A. and Toledo, D., A complex hyperbolic structure for moduli space of cubic surfaces, C. R. Acad. Sci., 326 (1998), 49-54.

[2] Allcock, D., New complex and quaternion-hyperbolic reflection groups, Duke Math. J., 103 (2000), 303-333.

[3] Deligne, P. and Mostow, G.D., Monodromy of hypergeometric functions and non-lattice integral monodromy, IHES Publ. Math., 63 (1986), 5-106.

[4] Dolgachev, I. and Ortland, D., Point sets in projective spaces and theta functions, Astereisque, 165 (1985).

[5] Gonzalez-Dies, G., Loci of curves which are prime Galois coverings of $\mathbb{P}^{1}$, Proc. London Math. Soc., 62 (1991), 469-489.

[6] Hunt, B., The Geometry of some special Arithmetic Quotients, LNM. 1637, Springer, 1996. 
[7] Igusa, J., Theta Functions, Springer, 1972.

[8] Mumford, D., Tata Lectures on Theta I, Birkhäuser, 1983.

[9] Matsumoto, K. and Terasoma, T., Theta constants associated to cubic three folds, Preprint (math.AG/0008024).

[10] Namba, M., Equivalence problem and automorphism groups of certain compact Riemann surfaces, Tsukuba J. Math., 5 (1981), 319-338.

[11] Picard, E., Sur les fonctions de deux variables indépendantes analogues aux fonctions modulaires, Acta Math., 2 (1883), 114-126.

[12] Shiga, H., On the representation of Picard modular function by $\theta$ constants I-II, Publ. RIMS, Kyoto Univ., 24 (1988), 311-360.

[13] Terada, T., Fonctions hypergéometriques $F_{1}$ et fonctions automorphes I, II, Math. Soc. Japan, 35 (1983), 451-475; 37 (1985), 173-185.

[14] Yoshida, M., Hypergeometric Functions, My Love, Vieweg, 1997.

[15] Yoshida, M., The real loci of the configuration space of six points on the projective line and a Picard modular 3-fold, Kumamoto J. Math., 11 (1998), 43-67. 